\title{
Exploring continuum structures with a pseudo-state basis
}

\author{
J. A. Lay, ${ }^{*}$ A. M. Moro, ${ }^{\dagger}$ and J. M. Arias ${ }^{\ddagger}$ \\ Departamento de FAMN, Facultad de Física, Universidad de Sevilla, Apdo. 1065, E-41080 Sevilla, Spain \\ J. Gómez-Camacho ${ }^{\S}$ \\ Departamento de FAMN, Facultad de Física, Universidad de Sevilla, Apdo. 1065, E-41080 Sevilla, Spain \\ and Centro Nacional de Aceleradores, Avda. Thomas A. Edison, E-41092, Sevilla, Spain \\ (Received 27 April 2010; revised manuscript received 7 July 2010; published 10 August 2010)
}

\begin{abstract}
The ability of a recently developed square-integrable discrete basis to represent the properties of the continuum of a two-body system is investigated. The basis is obtained performing a simple analytic local scale transformation to the harmonic oscillator basis. Scattering phase-shifts and the electric transition probabilities $B(E 1)$ and $B(E 2)$ have been evaluated for several potentials using the proposed basis. Both quantities are found to be in excellent agreement with the exact values calculated from the true scattering states. The basis has been applied to describe the projectile continuum in the ${ }^{6} \mathrm{He}$ scattering by ${ }^{12} \mathrm{C}$ and ${ }^{208} \mathrm{~Pb}$ targets at $240 \mathrm{MeV} /$ nucleon and the ${ }^{11} \mathrm{Be}$ scattering by ${ }^{12} \mathrm{C}$ at $67 \mathrm{MeV} /$ nucleon. The calculated breakup differential cross sections are found to be in very good agreement with the available experimental data for these reactions.
\end{abstract}

DOI: 10.1103/PhysRevC.82.024605

PACS number(s): 24.10.Eq, 25.10.+s, 25.45.De

\section{INTRODUCTION}

Quantum collisions involving weakly bound systems are known to be influenced by the coupling to the unbound states. In nuclear collisions, this was first evidenced in the pioneering study by Johnson and Soper [1], who recognized the relevance of the breakup channels to understand deuteroninduced reactions. In their survey, the deuteron continuum was represented by a single $s$ state. Later developments by Rawitscher [2] and Austern et al. [3] helped to introduce a more realistic representation of the continuum, leading to the development of the continuum-discretized coupled-channels (CDCC) method. This method reduces the many-body problem to an effective three-body problem and expands the full three-body wave function in a selected set of continuum wave functions of a given pair subsystem Hamiltonian. Projection of the Schrödinger equation onto the selected internal states gives rise to a set of coupled differential equations. The practical implementation of the method requires a discretization procedure, that is, an approximation of the two-body continuum spectrum by a finite and discrete representation. Although not strictly necessary, it is numerically advantageous to use for this representation a set of $\mathcal{L}^{2}$ (that is, square-integrable) functions. The standard method of continuum discretization consists in dividing the continuum into a set of energy or momentum intervals. For each interval, or bin, a representative wave function is constructed by superposition of the scattering states within the interval (the average method).

An alternative to the discretization method based on bins is the pseudo-state (PS) method, in which the wave functions describing the internal motion of the projectile are obtained

\footnotetext{
*lay@us.es

†moro@us.es

†ariasc@us.es

§gomez@us.es
}

as the eigenstates of the projectile Hamiltonian in a truncated basis of square-integrable functions. A variety of PS bases have been proposed in the literature for two-body continuum discretization [4-6] and, more recently, also for the three-body continuum [7-10].

In a recent work [6], we proposed a PS method based on a local scale transformation (LST) of the harmonic oscillator (HO) basis. The LST, adopted from a previous work of Karataglidis et al. [11], is such that it transforms the Gaussian asymptotic behavior into an exponential form, thus ensuring the correct asymptotic behavior for the bound wave functions. The accuracy of this transformed harmonic oscillator (THO) basis was tested for several reactions induced by deuterons and halo nuclei, showing an excellent agreement with the standard binning method and an improved convergence rate.

Due to their vanishing asymptotic behavior, it is not obvious that genuine continuum properties, such as the scattering phase-shifts, can be well described using square-integrable states. However, in this case one can make use of integral formulas, which require only the wave function within a finite region. Several prescriptions have been proposed in the literature to extract the phase shifts from continuumdiscretized states [12-14]. In this work, we make use of the stabilization method of Hazi and Taylor $[12,15,16]$ to show that the THO basis reproduces very well the exact phase-shifts. As an additional test of the quality of the THO basis, we calculate several transition probabilities and their associated sum rules. Finally, we apply the THO basis to calculate the breakup of the reactions ${ }^{6} \mathrm{He}+{ }^{12} \mathrm{C}$ and ${ }^{6} \mathrm{He}+{ }^{208} \mathrm{~Pb}$ at $240 \mathrm{MeV} /$ nucleon and ${ }^{11} \mathrm{Be}+{ }^{12} \mathrm{C}$ at $67 \mathrm{MeV} /$ nucleon, making use of the CDCC formalism. The calculations are compared with existing experimental data for these reactions.

The work is structured as follows. In Sec. II we review the THO method based on the parametric LST. In Sec. III we introduce an integral formula suitable for the calculation of scattering phase-shifts with PS functions. In Sec. IV we recall some useful formulas to evaluate the dipole and quadrupole 
transition probabilities from the scattering states and from the pseudo-states. In Sec. V these formulas are applied to study the continuum of the deuteron and ${ }^{6} \mathrm{He}$ nuclei. In Sec. VI the method is applied to the scattering of ${ }^{6} \mathrm{He}$ by ${ }^{12} \mathrm{C}$ and ${ }^{208} \mathrm{~Pb}$ at $240 \mathrm{MeV}$ per nucleon and by ${ }^{11} \mathrm{Be}+{ }^{12} \mathrm{C}$ at $67 \mathrm{MeV} /$ nucleon. Finally, in Sec. VII we summarize the main results of this work.

\section{THE ANALYTIC LST}

In this section, we briefly review the features of the PS basis used in this work. This basis was originally developed in Ref. [11] to describe the single-particle orbitals within a meanfield approach. In a later work [6], we adopted this method to discretize the continuum of a two-body system within the context of the continuum-discretized coupled-channels method.

The starting point is the HO basis in angular momentum representation. The radial part of the $n$th HO function for a given partial wave $\ell$ is here denoted as $\phi_{n, \ell}^{\mathrm{HO}}(s)$. These functions are orthogonal and constitute a complete set and, therefore, they can be used to expand the eigenstates (bound and unbound) of an arbitrary potential. For a finite well, the boundstate wave functions decay exponentially at large distances and hence the $\mathrm{HO}$ basis does not provide a suitable representation due to its Gaussian asymptotic form. A possible approach to overcome this limitation, while retaining the appealing properties of the HO basis, is to perform a LST that converts the Gaussian behavior into an exponential one [17,18]. This gives rise to the so-called transformed harmonic oscillator (THO) basis. We denote the radial part of these basis states as

$$
\phi_{n, \ell}^{\mathrm{THO}}(r)=\sqrt{\frac{d s}{d r}} \phi_{n, \ell}^{\mathrm{HO}}[s(r)] .
$$

Note that, by construction, the family of functions $\phi_{n, \ell}^{\mathrm{THO}}(r)$ is orthogonal and constitutes a complete set with the following normalization:

$$
\int_{0}^{\infty} d r\left|\phi_{n, \ell}^{\mathrm{THO}}(r)\right|^{2}=1
$$

Moreover, they decay exponentially at large distances, thus ensuring the correct asymptotic behavior for the bound wave functions. In practical calculations a finite set of functions (1) is retained, and the internal Hamiltonian of the projectile is diagonalized in this truncated basis with $N$ states, giving rise to a set of eigenvalues and their associated eigenfunctions, denoted, respectively, as $\left\{\varepsilon_{n}\right\}$ and $\left\{\varphi_{n, \ell}^{(N)}(r)\right\}(n=1, \ldots, N)$. As the basis size is increased, those eigenstates with negative energy will tend to the exact bound states of the system, while eigenstates with positive eigenvalues can be regarded as a finite representation of the unbound states.

With the aforementioned criterion, the LST is indeed not unique. In Ref. [19] the LST is defined in such a way that the first HO state is exactly transformed into the exact ground-state wave function. Therefore, by construction, this wave function is exactly recovered for any arbitrary size of the basis. In a more recent work [6] we adopted the parametric form of
Karataglidis et al. [11],

$$
s(r)=\frac{1}{\sqrt{2} b}\left[\frac{1}{\left(\frac{1}{r}\right)^{m}+\left(\frac{1}{\gamma \sqrt{r}}\right)^{m}}\right]^{\frac{1}{m}},
$$

which depends on the parameters $m, \gamma$, and the oscillator length $b$. Following Ref. [6], the oscillator length $b$ is treated as a variational parameter used to minimize the ground-state energy. Asymptotically, the function $s(r)$ behaves as $s(r) \sim$ $\frac{\gamma}{b} \sqrt{\frac{r}{2}}$ and hence the functions obtained by applying this LST to the HO basis behave at large distances as $\exp \left(-\gamma^{2} r / 2 b^{2}\right)$. Therefore, the ratio $\gamma / b$ can be related to an effective linear momentum, $k_{\text {eff }}=\gamma^{2} / 2 b^{2}$, which governs the asymptotic behavior of the THO functions; as the ratio $\gamma / b$ increases, the radial extension of the basis decreases and, consequently, the eigenvalues obtained upon diagonalization of the Hamiltonian in the THO basis tend to concentrate at higher energies. Therefore, $\gamma / b$ determines the density of PS as a function of the excitation energy. This property was used in Ref. [6] to determine a suitable value for the ratio $\gamma / b$ in scattering calculations. For a more quantitative measurement of the density of states we define the magnitude:

$$
\rho^{(N)}(k)=\sum_{n=1}^{N}\left\langle\varphi_{\ell}(k) \mid \varphi_{n, \ell}^{(N)}\right\rangle,
$$

with $\left|\varphi_{\ell}(k)\right\rangle$ denoting the scattering wave function for a momentum $k$.

With this definition the integral of the density with respect to the momentum is just the number of basis states, that is,

$$
\int_{0}^{\infty} d k \rho^{(N)}(k)=N
$$

regardless of the choice of the parameters of the LST.

In all the calculations presented in this work, the power $m$ is just taken as $m=4$. This was one of the choices made in Ref. [11] and, in fact, the authors of that work found a very weak dependence of the results on this parameter.

\section{EXTRACTING THE PHASE SHIFTS FROM THE THO BASIS}

The properties of the continuum states are completely determined by the phase shifts. In a two-body problem, the phase shifts are readily obtained from the asymptotics of the radial part of the wave function. Ignoring spins for simplicity, the radial part corresponding to a partial wave $\ell$ can be written at large distances as

$$
\varphi_{\ell}(k, r) \rightarrow \sqrt{\frac{2}{\pi}}\left[\cos \delta_{\ell}(k) F_{\ell}(k r)+\sin \delta_{\ell}(k) G_{\ell}(k r)\right],
$$

where $F_{\ell}$ and $G_{\ell}$ are the regular and irregular Coulomb functions. If the potential is real, the functions $\varphi_{\ell}$ as well as the phase shifts $\delta_{\ell}$ are also real.

Equation (6) cannot be applied to PS functions to extract the phase shifts, because these functions vanish asymptotically. However, the phase shifts can be also obtained from integral expressions, which require only the interior part of the wave 
functions. Here, we make use of the integral formula proposed by Hazi and Taylor $[12,16]$, who applied this formula to extract the phase shifts in a one-dimensional scattering problem using a harmonic oscillator representation. We have generalized this formula to three-dimensional cases. The formula so obtained reads

$$
\tan \delta_{\ell}(k)=-\frac{\int_{0}^{\infty} \varphi_{\ell}(k, r)[E-H] f(r) F_{\ell}(k r) d r}{\int_{0}^{\infty} \varphi_{\ell}(k, r)[E-H] f(r) G_{\ell}(k r) d r} .
$$

This formula can be derived following the same arguments outlined in Ref. [12] for the one-dimensional case. We note that, if the exact wave functions are used for $\varphi_{\ell}(k, r)$, this expression becomes an alternative to Eq. (6) to calculate the exact phase shifts. The function $f(r)$ appearing in Eq. (7) verifies the following properties:

$$
f(r) \stackrel{r \rightarrow \infty}{\longrightarrow} 1 ; \quad f(0)=f^{\prime}(0)=0 .
$$

Following Ref. [12], we adopt the explicit form $f(r)=1-$ $\exp \left(-\beta r^{2}\right)$, with $\beta>0$. The aim of this function $f(r)$ is to avoid evaluating the function $G_{\ell}(k r)$ at the origin, where it becomes singular. Therefore the parameter $\beta$ should be small enough to make $f(r) \approx 0$ for distances of the order of the nuclear range. In the cases studied here, we have chosen $\beta=$ $0.01 \mathrm{fm}^{-2}$.

\section{ELECTRIC TRANSITION PROBABILITIES IN THE PSEUDO-STATE BASIS}

The accuracy of the PS basis to represent the continuum can be studied by comparing the ground-state to continuum transition probability due to a given operator. Here we consider the important case of the electric dissociation of the initial nucleus $a$ into the fragments $b+c$. This involves a matrix element between a bound state (typically the ground state) and the continuum states.

The electric transition probability between two bound states, $\left|\left(\ell_{i} s\right) j_{i}\right\rangle$ and $\left|\left(\ell_{f} s\right) j_{f}\right\rangle$ (assumed here to be normalized to unity), is given by the reduced matrix element

$$
B(E \lambda ; i \rightarrow f)=\frac{2 j_{f}+1}{2 j_{i}+1}\left|\left\langle\left(\ell_{f} s\right) j_{f}\|\mathcal{M}(E \lambda)\|\left(\ell_{i} s\right) j_{i}\right\rangle\right|^{2},
$$

where $\mathcal{M}$ is the multipole operator,

$$
\mathcal{M}(E \lambda \mu)=Z_{\mathrm{eff}}^{(\lambda)} e r^{\lambda} Y_{\lambda \mu}(\hat{r}),
$$

with the effective charge

$$
Z_{\mathrm{eff}}^{(\lambda)}=Z_{b}\left(\frac{m_{c}}{m_{b}+m_{c}}\right)^{\lambda}+Z_{c}\left(-\frac{m_{b}}{m_{b}+m_{c}}\right)^{\lambda} .
$$

In the case of a transition to a continuum of states, $\left|k\left(\ell_{f} s\right) j_{f}\right\rangle$, the preceding definition is replaced by (see, e.g., Ref. [20])

$$
\begin{aligned}
\frac{d B(E \lambda)}{d \varepsilon}= & \frac{2 j_{f}+1}{2 j_{i}+1} \frac{\mu_{b c} k}{(2 \pi)^{3} \hbar^{2}} \\
& \times\left|\left\langle k\left(\ell_{f} s\right) j_{f}\|\mathcal{M}(E \lambda)\|\left(\ell_{i} s\right) j_{i}\right\rangle\right|^{2},
\end{aligned}
$$

with $k=\sqrt{2 \mu_{b c} \varepsilon} / \hbar$. Note that the extra factor appearing in Eq. (12) with respect to Eq. (9) is consistent with the convention $\left\langle k(\ell s) j \mid k^{\prime}(\ell s) j\right\rangle=\delta\left(k-k^{\prime}\right)$ and the asymptotic behavior of Eq. (6).

In the calculations presented in this work, we ignore for simplicity the internal spins of the clusters and hence $s=0$, $j_{i}=\ell_{i}$, and $j_{f}=\ell_{f}$. In addition, we consider only transitions from the ground state, so $\left\langle\mathbf{r} \mid\left(\ell_{i} s\right) j_{i}\right\rangle=\varphi_{\text {g.s. }}$. $(\mathbf{r})$ (where the index $\ell_{i}$ is omitted for shortness). This reduces Eq. (12) to

$$
\frac{d B(E \lambda)}{d \varepsilon}=\frac{2 \ell_{f}+1}{2 \ell_{i}+1} \frac{\mu_{b c} k}{(2 \pi)^{3} \hbar^{2}}\left|\left\langle\varphi_{\ell_{f}}(k)|| \mathcal{M}(E \lambda)|| \varphi_{\text {g.s. }}\right\rangle\right|^{2} .
$$

The reduced matrix element is given by

$$
\left\langle\varphi_{\ell_{f}}(k)\|\mathcal{M}(E \lambda)\| \varphi_{\text {g.s. }}\right\rangle=\frac{4 \pi}{k} Z_{\text {eff }} e D_{\ell_{i}, \ell_{f}}^{(\lambda)} R_{\ell_{i}, \ell_{f}}^{\lambda}(k),
$$

with $D_{\ell_{i}, \ell_{f}}^{(\lambda)}$ being a geometric factor [20] and $R_{\ell_{i}, \ell_{f}}^{\lambda}(k)$ the radial integral

$$
R_{\ell_{i}, \ell_{f}}^{\lambda}(k)=\int_{0}^{\infty} d r \varphi_{\ell_{f}}(k, r) r^{\lambda} \varphi_{\text {g.s. }}(r) .
$$

Using a finite basis, one may calculate only discrete values for the transition probability. According to Eq. (9), the $B(E \lambda)$ between the ground state and the $n$th PS is given by

$$
B^{(N)}(E \lambda ; \text { g.s. } \rightarrow n)=\frac{2 \ell_{f}+1}{2 \ell_{i}+1}\left|\left\langle\varphi_{n, \ell_{f}}^{(N)} \| \mathcal{M}(E \lambda)|| \varphi_{\text {g.s. }}\right\rangle\right|^{2} .
$$

To relate this discrete representation with the continuous distribution (13) one may use the simple approximation

$$
\left.\frac{d B(E \lambda)}{d \varepsilon}\right|_{\varepsilon=\varepsilon_{n}} \simeq \frac{1}{\Delta_{n}} B^{(N)}(E \lambda ; \text { g.s. } \rightarrow n),
$$

where $\Delta_{n}=\left(\varepsilon_{n+1}-\varepsilon_{n-1}\right) / 2$ is an estimate for the energy width of the $n$th PS. This expression provides the $B(E \lambda)$ values only for the PS eigenvalues $\varepsilon_{n}$.

Alternatively, one may derive a continuous approximation to Eq. (13) by introducing the identity in the truncated PS basis, that is,

$$
I_{\ell}^{N}=\sum_{n=1}^{N}\left|\varphi_{n, \ell}^{(N)}\right\rangle\left\langle\varphi_{n, \ell}^{(N)}\right| .
$$

For $N \rightarrow \infty$ this expression tends to the exact identity operator for the Hilbert space spanned by the eigenfunctions of the considered Hamiltonian. By inserting Eq. (18) into the exact expression (13), we obtain the approximated continuous distribution:

$$
\begin{aligned}
\frac{d B(E \lambda)}{d \varepsilon} \simeq & \frac{2 \ell_{f}+1}{2 \ell_{i}+1} \frac{\mu_{b c} k}{(2 \pi)^{3} \hbar^{2}} \\
& \times\left|\sum_{n=1}^{N}\left\langle\varphi_{\ell}(k) \mid \varphi_{n, \ell}^{(N)}\right\rangle\left\langle\varphi_{n, \ell}^{(N)}|| \mathcal{M}(E \lambda)|| \varphi_{g . s .}\right\rangle\right|^{2} .
\end{aligned}
$$


To test the accuracy of the THO basis to describe the continuum, we calculate also the following magnitudes:

(i) The non-energy-weighted sum rule (NEWSR):

$$
\begin{aligned}
\mathrm{NEWSR} & \equiv \int d \varepsilon \frac{d B(E \lambda)}{d \varepsilon} \\
& =\frac{2 \ell_{f}+1}{2 \ell_{i}+1}\left(D_{\ell_{i}, \ell_{f}}^{(\lambda)}\right)^{2}\left\langle r^{2 \lambda}\right\rangle_{\text {g.s. }},
\end{aligned}
$$

with $\left\langle r^{2 \lambda}\right\rangle_{\text {g.s. }} \equiv\left\langle\varphi_{\text {g.s. }}\left|r^{2 \lambda}\right| \varphi_{\text {g.s. }}\right\rangle$.

(ii) The energy-weighted sum rule (EWSR):

$$
\begin{aligned}
\mathrm{EWSR} & \equiv \int d \varepsilon \frac{d B(E \lambda)}{d \varepsilon}\left(\varepsilon-\varepsilon_{\text {g.s. }}\right) \\
& =\frac{\hbar^{2}}{2 \mu_{b c}} \lambda(2 \lambda+1) \frac{2 \ell_{f}+1}{2 \ell_{i}+1}\left(D_{\ell_{i}, \ell_{f}}^{(\lambda)}\right)^{2}\left\langle r^{2 \lambda-2}\right\rangle_{\text {g.s. }}
\end{aligned}
$$

(iii) The energy-inverse-weighted integral (or polarizability):

$$
\alpha \equiv \frac{8 \pi}{9} \int d \varepsilon \frac{1}{\left(\varepsilon-\varepsilon_{\text {g.s. }}\right)} \frac{d B(E \lambda)}{d \varepsilon} .
$$

Due to their respective weight factors, the EWSR and the polarizability are useful quantities to test the accuracy of the basis to describe high-energy and low-energy parts of the spectrum, respectively. Note that the closed expression for the EWSR is only valid for angular-momentum-independent Hamiltonians. We note also that there is no closed expression for the polarizability, but to calculate this quantity with the desired accuracy, as well as the EWSR for angular-momentumdependent Hamiltonians, one can directly evaluate Eq. (22) using the exact continuum states integrated up to a sufficiently high excitation energy.

\section{APPLICATION TO NUCLEAR STRUCTURE}

\section{A. Application to the deuteron}

As an illustration of the expressions derived in the preceding sections, we first consider the case of the $p$ - $n$ system with a central potential. Following Ref. [21], the interaction between the proton and the neutron is parametrized in terms of the Poeschl-Teller potential,

$$
V_{\mathrm{pn}}(r)=-\frac{V_{0}}{\cosh (a r)^{2}},
$$

with $V_{0}=102.706 \mathrm{MeV}$ and $a=0.9407 \mathrm{fm}^{-1}$. With these values, the ground-state energy is $2.2245 \mathrm{MeV}$, in agreement with the experimental value.

The oscillator length was chosen to minimize the groundstate energy obtained upon diagonalization of the Hamiltonian in a small THO basis. This yields the value $b=1.5 \mathrm{fm}$. Once the value of $b$ is fixed, the ratio $\gamma / b$ determines the extension of the PS eigenstates; increasing the value of $\gamma$ reduces the radial extension and pushes the eigenvalues to higher energies. This is better seen in terms of the density of states, defined according to Eq. (4). This magnitude is plotted in Fig. 1 for the $\ell=0$ continuum, using a basis of $N=30$ states, and three different choices of $\gamma$, namely, $\gamma=1 \mathrm{fm}^{1 / 2}, 2.48 \mathrm{fm}^{1 / 2}$, and $5 \mathrm{fm}^{1 / 2}$. It is seen that small values of $\gamma$ (which correspond

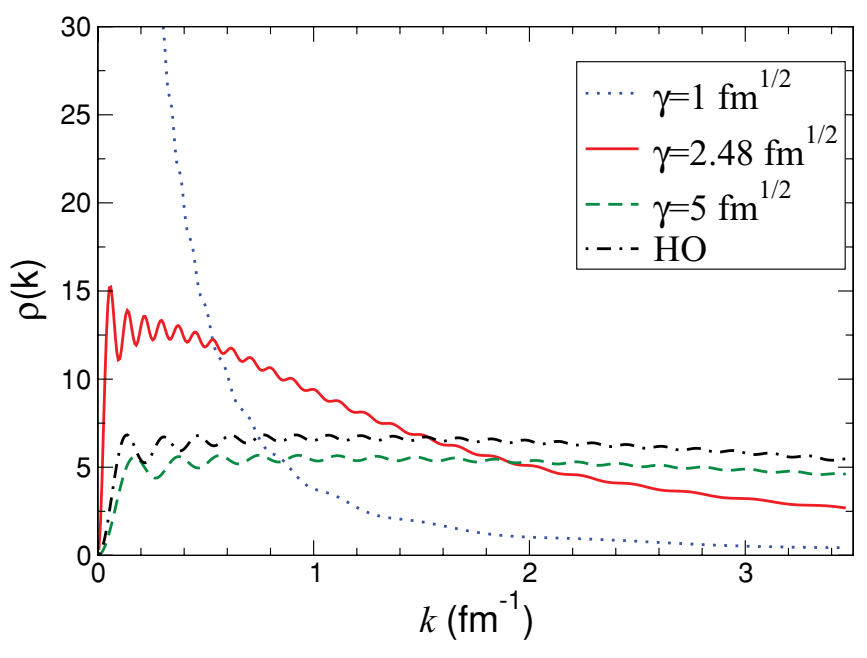

FIG. 1. (Color online) Density of states for the $\ell=0$ continuum obtained with a THO basis with $N=30$ states, defined according to Eq. (4) for different choices of the $\gamma$ parameter. The dot-dashed curve is the density obtained with a $\mathrm{HO}$ basis with 30 states and $b=2 \mathrm{fm}$. See text for details.

to an extended THO basis in configuration space) produce a fine description of the continuum at low energy. This is useful, for example, to study Coulomb breakup. Increasing the value of $\gamma$ will decrease the density of states at low energies, which is compensated by an increase of the density at higher excitation energies. The most suitable choice for this parameter will depend on the problem at hand, depending on the energy region of interest. We emphasize, however, that the dependence on $\gamma$ is not critical and, in the applications shown here, different values of $\gamma$ converge to the same results for sufficiently large bases. For comparison, we include also in Fig. 1 the density obtained with a $\mathrm{HO}$ basis with $N=30$ states and $b=2.0 \mathrm{fm}$, which minimizes the ground-state energy for the HO basis.

We next consider the scattering phase-shifts. These are displayed in Fig. 2 as a function of the relative $p-n$ energy. The top, middle, and bottom panels correspond to the $s, p$, and $d$ waves. The solid line is the calculation using the asymptotics of the exact scattering states, whereas the circles represent the calculation obtained with Eq. (7), using a THO basis with $N=30$ states. The LST was generated with the parameters $b=1.5 \mathrm{fm}$ and $\gamma=2.48 \mathrm{fm}^{1 / 2}$. In the three cases, we find an excellent agreement between the exact and the approximate phase-shifts in the whole energy range.

Note that the calculated phase shifts are consistent with the Levinson theorem (see, e.g., Ref. [22]), which establishes that the phase shift at zero energy is given by $\delta_{\ell}(0)=n \pi$, where $n$ is the number of bound states for the partial wave $\ell$. So, because the $s$ wave supports a bound state (the deuteron), we have $\delta_{0}(0)=\pi$, whereas for $\ell=1$ and $\ell=2$ we have $\delta_{1}(0)=\delta_{2}(0)=0$.

We now consider the electric transition probabilities, $B(E 1)$ and $B(E 2)$. These are shown in Fig. 3. The solid line corresponds to the calculation using the scattering states Eq. (13), the solid circles correspond to the discrete approximation using the THO basis Eq. (17), and the dashed line is the calculation obtained folding the discrete distribution with the continuum states [Eq. (19)]. Both the discrete and folded approximations 

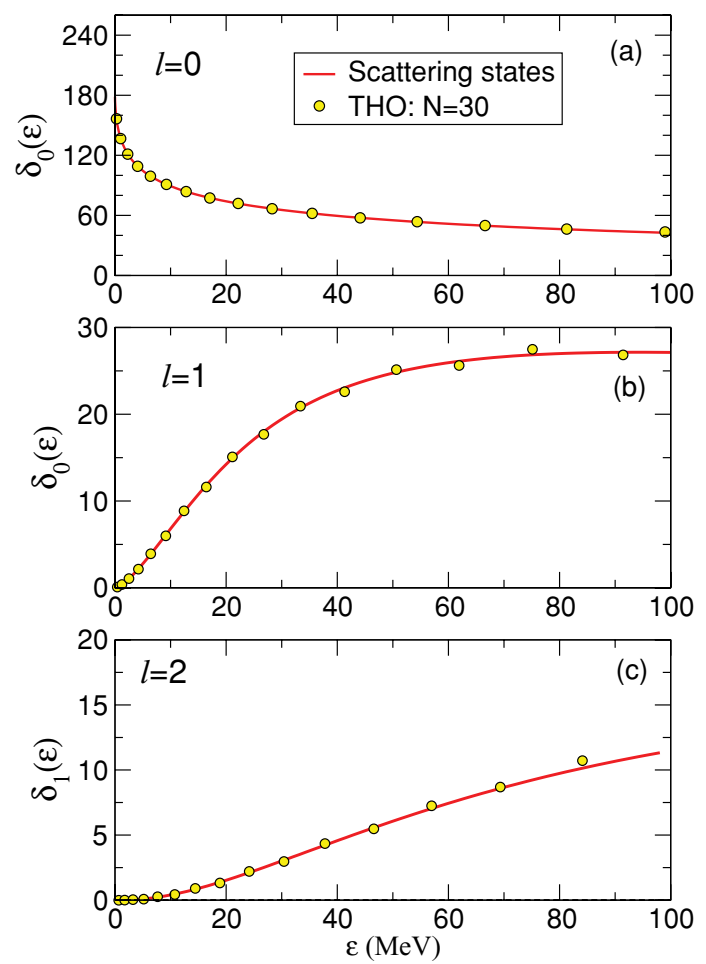

FIG. 2. (Color online) Phase shifts for the deuteron system as a function of the relative $p-n$ energy. The upper, middle, and bottom panels correspond to $\ell=0,1$, and 2 continuum states, respectively. See text for details.
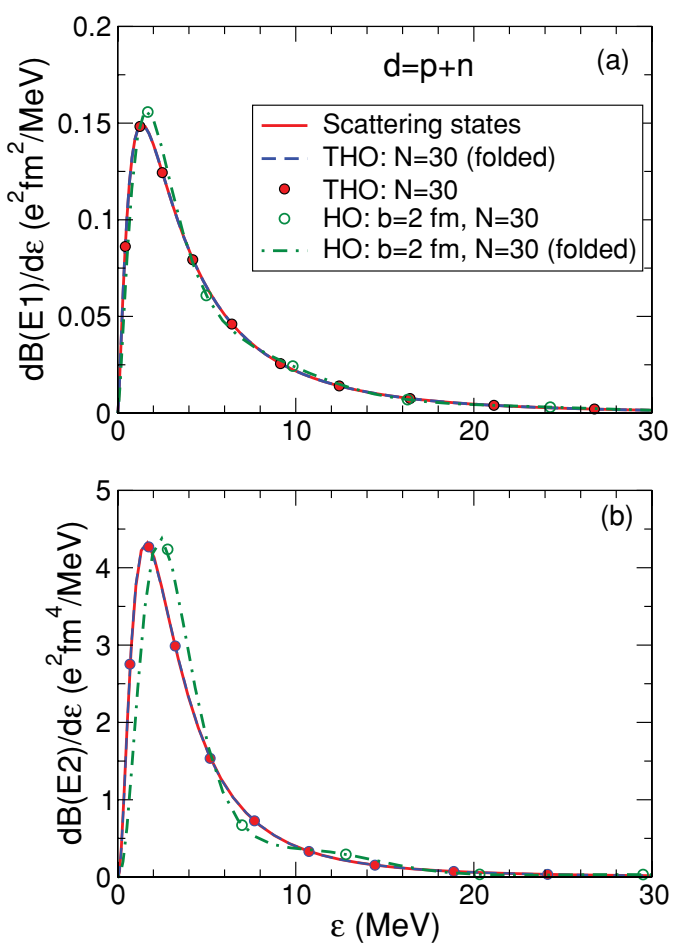

FIG. 3. (Color online) Electric transition probabilities for the $d=$ $p+n$ system. The top and bottom panels correspond to $\lambda=1$ and $\lambda=2$ transitions, respectively. See text for details.
TABLE I. Convergence of the ground-state energy and the total $B(E 1)$ and $B(E 2)$ transition probabilities for the deuteron case.

\begin{tabular}{|c|c|c|c|c|c|c|}
\hline \multirow[t]{2}{*}{$N$} & \multicolumn{2}{|c|}{$\varepsilon_{\text {g.s. }}(\mathrm{MeV})$} & \multicolumn{2}{|c|}{$\begin{array}{c}\text { Total } \\
B(E 1)\left(\mathrm{e}^{2} \mathrm{fm}^{2}\right)\end{array}$} & \multicolumn{2}{|c|}{$\begin{array}{c}\text { Total } \\
B(E 2)\left(\mathrm{e}^{2} \mathrm{fm}^{4}\right)\end{array}$} \\
\hline & $\mathrm{HO}$ & THO & $\mathrm{HO}$ & THO & $\mathrm{HO}$ & THO \\
\hline 0 & -2.1570 & -2.2 & 0.81380 & 86 & 14. & 17.9729 \\
\hline 20 & -2.2201 & -2.2245 & 0.86200 & 0.87129 & 19.1257 & 20.7938 \\
\hline 30 & -2.2237 & -2.2245 & 0.86926 & 0.87136 & 20.2919 & 20.8297 \\
\hline 10 & -2.2241 & -2.2245 & 0.87079 & 0.87136 & 20.6422 & 20.8297 \\
\hline 50 & -2.2242 & -2.2245 & 0.87119 & 0.87136 & 20.7591 & 20.8297 \\
\hline 60 & -2.2243 & -2.2245 & 0.87130 & 0.87136 & 20.8015 & 20.8297 \\
\hline 70 & -2.2243 & -2.2245 & 0.87134 & 0.87136 & 20.8178 & 20.8297 \\
\hline Exact & \multicolumn{2}{|c|}{-2.2245} & \multicolumn{2}{|c|}{0.87136} & \multicolumn{2}{|c|}{20.8297} \\
\hline
\end{tabular}

show an excellent agreement with the exact distribution. We include also the calculation using the $\mathrm{HO}$ basis with $N=30$ states and $b=2.0 \mathrm{fm}$ (open circles and dot-dashed line). Both the $B(E 1)$ and $B(E 2)$ distributions depart significantly from the exact distributions. In addition, the HO basis produces a small density of states at low energy, which might be a drawback for scattering calculations.

In Tables I and II we present the convergence of the groundstate energy and the $E 1$ and $E 2$ sum rules with respect to the basis size. The last row lists the exact values obtained with the closed expressions of Eqs. (20) and (21). It is seen that with a moderately small basis one obtains a very good convergence to the exact values. For comparison, in these tables we include also the calculations using the HO basis. From the quoted numbers, it is clear that the convergence rate is much faster for the THO basis.

It is worth noting that, despite the simple Hamiltonian adopted in this work for the $p$ - $n$ system, the calculated polarizability is fully consistent with the experimental value, $\alpha_{\text {exp }}=0.61 \pm 0.04$, quoted in Ref. [23].

\section{B. Application to ${ }^{6} \mathrm{He}$}

We now consider a situation in which more complicated continuum structures are present, such as resonances. For this purpose, we take the ${ }^{6} \mathrm{He}$ nucleus, treated as a two-body

TABLE II. Convergence of the polarizability and EWSR for the deuteron case.

\begin{tabular}{|c|c|c|c|c|}
\hline \multirow[t]{2}{*}{$N$} & \multicolumn{2}{|c|}{$\alpha(\mathrm{fm})^{3}$} & \multicolumn{2}{|c|}{$\begin{array}{c}\text { Energy-weighted } \\
B(E 1)\left(\mathrm{e}^{2} \mathrm{fm}^{2} \mathrm{MeV}\right)\end{array}$} \\
\hline & $\mathrm{HO}$ & THO & $\mathrm{HO}$ & THO \\
\hline 10 & 0.413988 & 0.515397 & 8.605586 & 8.102094 \\
\hline 20 & 0.557520 & 0.619350 & 7.742321 & 7.442755 \\
\hline 30 & 0.599683 & 0.620899 & 7.526588 & 7.437322 \\
\hline 40 & 0.613204 & 0.620922 & 7.466005 & 7.434683 \\
\hline 50 & 0.617923 & 0.620931 & 7.446418 & 7.433209 \\
\hline 60 & 0.620109 & 0.620936 & 7.439133 & 7.432331 \\
\hline 70 & 0.620395 & 0.620940 & 7.435989 & 7.431757 \\
\hline Exact & \multicolumn{2}{|c|}{0.620953} & \multicolumn{2}{|c|}{7.429937} \\
\hline
\end{tabular}



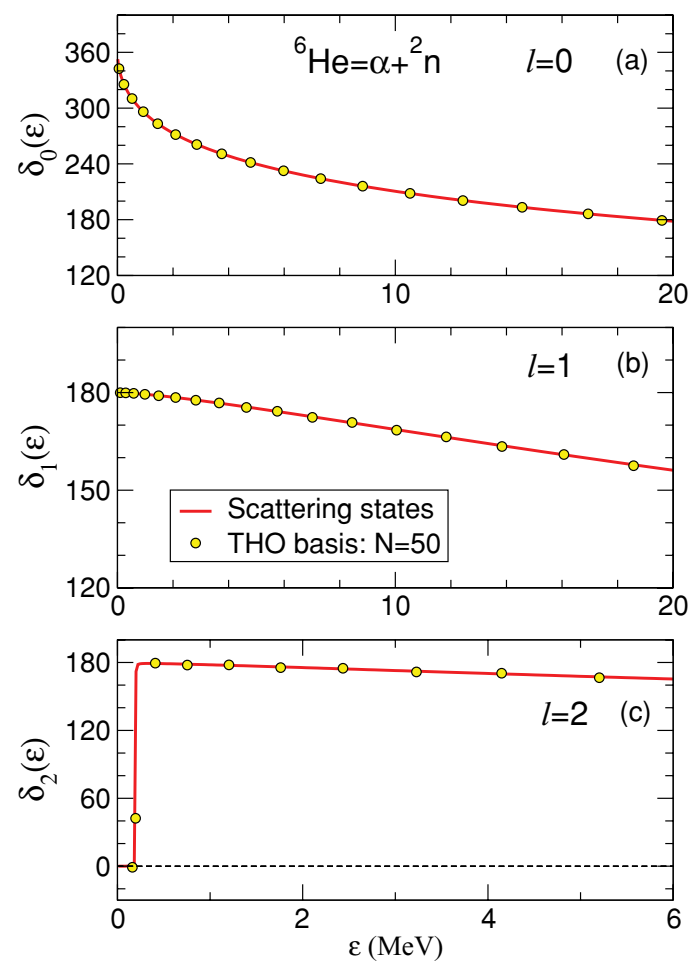

FIG. 4. (Color online) Phase shifts for the ${ }^{6} \mathrm{He}$ system as a function of the relative $\alpha+2 n$ energy. The top, middle, and bottom panels correspond to $\ell=0,1$, and 2 continuum states, respectively. See text for details.

system $\alpha+2 n$. Following Ref. [24], the interaction between the two clusters is described with a Woods-Saxon shape, with $R=1.9 \mathrm{fm}$ and $a=0.39 \mathrm{fm}$. For $\ell=0$ states, the depth of this potential is adjusted to give the effective separation energy of $1.6 \mathrm{MeV}$ between the two clusters. It was shown in Ref. [24] that using this effective binding energy, instead of the two-neutron separation energy $\left(S_{2 n}=0.97 \mathrm{MeV}\right)$, provides a more realistic description of the ground-state wave function. For $\ell=2$, the intercluster potential is adjusted to yield a resonance at an excitation energy of $E_{x}=1.8 \mathrm{MeV}$. For $\ell=1$, we simply took the depth found for $\ell=0$. A THO basis with $N=50$ states was used, and the LST was generated with the parameters $b=1 \mathrm{fm}$ and $\gamma=1.89 \mathrm{fm}^{1 / 2}$.

The $s-, p-$, and $d$-wave phase shifts are displayed in Fig. 4 as a function of the $\alpha+2 n$ relative energy, $\varepsilon$. For $\ell=2$, the energy scale has been restricted to the energy interval $\varepsilon=$ 0-6 MeV to emphasize the region of the resonance. Again, we find a perfect agreement between the exact (solid line) and the approximate (circles) phase shifts in the whole energy range. Note that, in this case, the $s$-wave potential supports two bound states, the Pauli forbidden $1 S$ state and the $2 S$ ground state. Consistently, the phase shift at zero energy is given by $\delta_{0}(0)=2 \pi$. Analogously, for $\ell=1$ the phase shift tends to $\pi$, due to the presence of a (Pauli forbidden) bound state in this partial wave. Finally, for $\ell=2$ no bound states are supported by this potential and, therefore, $\delta_{2}(0)=0$. For the $d$-wave, the phase shift crosses abruptly $\pi / 2$ at $\varepsilon=0.20 \mathrm{MeV}$, reflecting the presence of a narrow $2^{+}$resonance. Interestingly, this behavior is also observed in the THO basis, where there

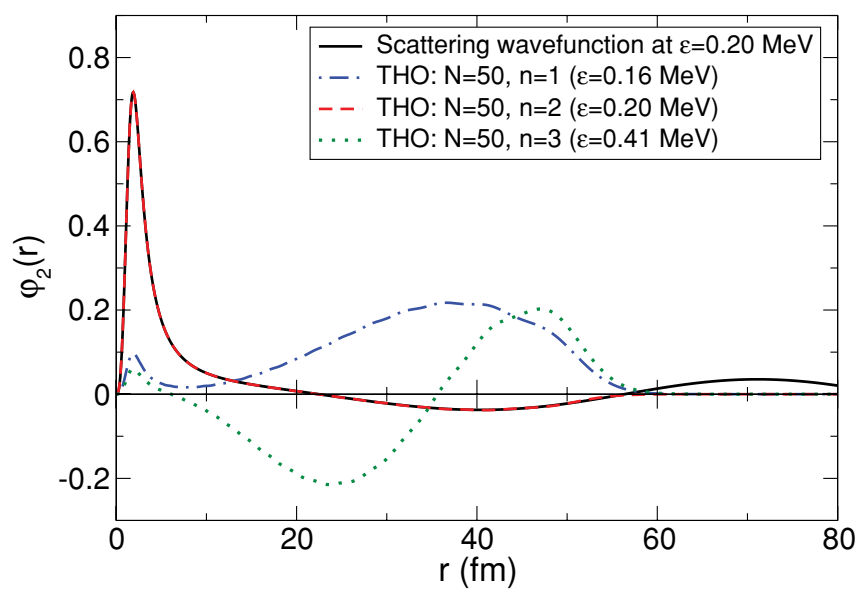

FIG. 5. (Color online) Radial part of the continuum wave function at the resonance for the ${ }^{6} \mathrm{He}$ system.

is a PS that appears exactly at the nominal energy of the resonance. It is then tempting to conclude that this PS will carry most of the character of the resonance, and in fact this is confirmed in Fig. 5, where we show the radial part of the exact scattering wave function, calculated at the energy of the resonance (solid line) along with the radial part of the PS eigenstate that appears at the energy of the resonance (dashed line). The former has been arbitrary normalized so that the two wave functions coincide at the maximum. It is seen that both wave functions are very similar up to very large distances. For comparison, we have included also the PS eigenstates associated with the eigenvalues just below (dotted-dashed line) and above (dotted line) the resonant one. They are very different from the scattering wave function at the resonance. In particular, it can be seen that a significant part of the norm of the resonant wave function is concentrated in the interior, as expected for a resonance, whereas for the nonresonant PS eigenstates, the probability in the interior is very small. This leads to the conclusion that in this case the character of the resonance is very well described by a single PS eigenstate. Indeed, if the basis is increased, the resonant character will be distributed among several PS. These results clearly show that a distinctive feature of the continuum such as the resonant structures are very well accounted for by the PS basis, despite its wrong asymptotic behavior.

In Fig. 6 we compare the $E 1$ and $E 2$ transition probabilities obtained from the scattering states by means of Eq. (13) (solid line), with the approximate distributions calculated with the THO basis. The circles correspond to the discrete expression of Eq. (17), whereas the dashed line is the smooth distribution obtained with Eq. (19). Both the discrete and smooth distributions are in excellent agreement with the exact distribution.

In Table III we display the convergence of the ground-state energy and the $E 1$ and $E 2$ sum rules with respect to the basis size. As in the deuteron case, both observables converge very fast to their exact value, given by the sum rule of Eq. (20).

Finally, in Table IV we present the convergence of the integrated-energy-weighted $B(E 1)$ and the polarizability. For 

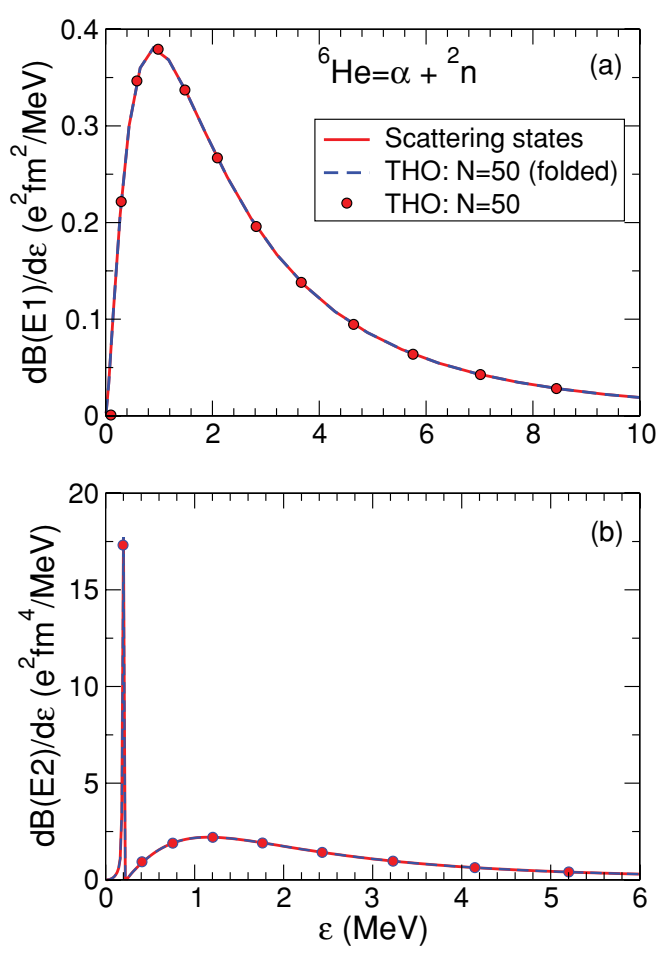

FIG. 6. (Color online) Electric transition probabilities for the ${ }^{6} \mathrm{He}=\alpha+2 n$ system. The top and bottom panels correspond to $\lambda=1$ and $\lambda=2$ transitions, respectively. See text for details.

the former, we cannot use the closed expression of Eq. (21), because the ${ }^{6} \mathrm{He}$ Hamiltonian depends on the angular momentum. The exact value listed in this Table corresponds to the explicit calculation using the scattering states up to a high excitation energy. The same holds for the polarizability, because for this observable there is no closed expression.

\section{APPLICATIONS TO NUCLEAR REACTIONS}

\section{A. Application to the reactions ${ }^{6} \mathrm{He}+{ }^{12} \mathrm{C}$ and ${ }^{6} \mathrm{He}+{ }^{208} \mathrm{~Pb}$ at $240 \mathrm{MeV} /$ nucleon}

The THO basis considered in this work is intended to provide a suitable discrete representation of the continuum spectrum of a loosely bound system, which can be useful for

TABLE III. Convergence of the ground-state energy and the total $B(E 1)$ and $B(E 2)$ transition probabilities for the ${ }^{6} \mathrm{He}=\alpha+2 n$ system.

\begin{tabular}{lccc}
\hline \hline$N$ & $\begin{array}{c}\varepsilon_{\text {g.s. }} \\
(\mathrm{MeV})\end{array}$ & $\begin{array}{c}\text { Total } B(E 1) \\
\left(\mathrm{e}^{2} \mathrm{fm}^{2}\right)\end{array}$ & $\begin{array}{c}\text { Total } B(E 2) \\
\left(\mathrm{e}^{2} \mathrm{fm}^{4}\right)\end{array}$ \\
\hline 10 & -1.5913 & 1.3538 & 8.5524 \\
20 & -1.5999 & 1.3854 & 9.7471 \\
30 & -1.5999 & 1.3855 & 9.7471 \\
40 & -1.5999 & 1.3855 & 9.7471 \\
50 & -1.6000 & 1.3855 & 9.7471 \\
80 & -1.6000 & 1.3855 & 9.7471 \\
Exact & $-\mathbf{1 . 6 0 0 0}$ & $\mathbf{1 . 3 8 5 5}$ & $\mathbf{9 . 7 4 7 \mathbf { 1 }}$ \\
\hline \hline
\end{tabular}

TABLE IV. Convergence of the polarizability and energyweighted $B(E 1)$ for the ${ }^{6} \mathrm{He}=\alpha+2 n$ system.

\begin{tabular}{lcc}
\hline \hline$N$ & $\begin{array}{c}\alpha \\
\left(\mathrm{fm}^{3}\right)\end{array}$ & $\begin{array}{c}\text { Total-energy-weighted } B(E 1) \\
\left(\mathrm{e}^{2} \mathrm{fm}^{2} \mathrm{MeV}\right)\end{array}$ \\
\hline 20 & 1.8652 & 6.5623 \\
30 & 1.8746 & 6.5464 \\
40 & 1.8750 & 6.5438 \\
50 & 1.8752 & 6.5425 \\
60 & 1.8753 & 6.5418 \\
70 & 1.8753 & 6.5412 \\
120 & 1.8755 & 6.5401 \\
Exact & $\mathbf{1 . 8 7 5 6}$ & $\mathbf{6 . 5 3 9 3}$ \\
\hline
\end{tabular}

scattering calculations within the CDCC method [3]. As a test case, we apply the THO basis to the reactions ${ }^{6} \mathrm{He}+{ }^{12} \mathrm{C}$ and ${ }^{6} \mathrm{He}+{ }^{208} \mathrm{~Pb}$ at $240 \mathrm{MeV} /$ nucleon. These reactions were measured by Aumann et al. [25] at the GSI facility with the aim of extracting information on the ${ }^{6} \mathrm{He}$ nucleus. The breakup of ${ }^{6} \mathrm{He}$ on ${ }^{208} \mathrm{~Pb}$ was already analyzed using the $\mathrm{CDCC}$ method with the THO basis in Ref. [6], showing an excellent agreement with the binning discretization method for the modulus of the breakup $S$ matrix. In this work, we extend the analysis of Ref. [6] to compare with the data of Ref. [25]. In particular, we consider the exclusive breakup differential cross section as a function of the excitation energy of the projectile $d \sigma / d E_{x}$. In Ref. [25], this observable was obtained by reconstructing the kinematics of the ${ }^{6} \mathrm{He}$ c.m. from the measured momenta of the outgoing fragments $\left({ }^{4} \mathrm{He}+n+n\right)$ and integrating up to a laboratory scattering angle of $80 \mathrm{mrad}$. To obtain this observable in our calculations, we first construct the double-differential cross section $d \sigma / d \Omega d E_{x}$ from the breakup $S$ matrices. In principle, the breakup $S$ matrix is a continuous function of the asymptotic momentum $k$. However, within a PS representation of the continuum, only discrete values of the $S$ matrix are obtained, corresponding to the eigenvalues $\varepsilon_{n}$. A continuous breakup $S$ matrix can be obtained from the solution of the coupled equations following the procedure used in Refs. [4,6,26], in which the discrete $S$ matrices are folded with the exact scattering states, similarly to what was done with the $B(E \lambda)$ distribution in Eq. (19), that is,

$$
S_{f: i}(k) \approx \sum_{n=1}^{N}\left\langle\varphi_{\ell_{f}}(k) \mid \varphi_{n, \ell_{f}}^{(N)}\right\rangle \hat{S}_{n: i}^{(N)}\left(k_{n}\right),
$$

where $\hat{S}_{n: i}^{(N)}\left(k_{n}\right)$ are the discrete $S$ matrix elements resulting from the solution of the coupled-channels equations using a PS basis with $N$ states. The subscripts $i, n$, and $f$ denote the channels $\left\{\varphi_{\text {g.s. }} ; L_{i}, \ell_{i}, J\right\},\left\{\varphi_{n, \ell_{f}}^{(N)} ; L_{f}, \ell_{f}, J\right\}$, and $\left\{\varphi_{\ell_{f}}(k) ; L_{f}, \ell_{f}, J\right\}$, respectively, where $L_{i}\left(L_{f}\right)$ is the initial (final) orbital angular momentum for the projectile-target relative motion and $J$ is the total angular momentum of the system.

We first consider the $\mathrm{Pb}$ target. For the $2 n-\alpha$ potential and the parameters of the THO basis we keep the same values of the preceding section. These parameters were found to provide an appropriate distribution of eigenstates for the ${ }^{6} \mathrm{He}+$ ${ }^{208} \mathrm{~Pb}$ reaction at $240 \mathrm{MeV} /$ nucleon [6]. The $\alpha+{ }^{208} \mathrm{~Pb}$ and 
$2 n+{ }^{208} \mathrm{~Pb}$ interactions are needed to generate the diagonal and nondiagonal coupling potentials of the CDCC equations. For the $\alpha+{ }^{208} \mathrm{~Pb}$ interaction we have adopted the first optical potential used in Ref. [27]. For the $2 n+{ }^{208} \mathrm{~Pb}$ interaction, we used the following single-folding model:

$U(\mathbf{R})=\int \rho_{n n}\left(r_{n n}\right)\left\{U_{n}\left(\mathbf{R}+\frac{\mathbf{r}_{n n}}{2}\right)+U_{n}\left(\mathbf{R}-\frac{\mathbf{r}_{n n}}{2}\right)\right\} d \mathbf{r}_{n n}$,

where $U_{n}$ is the neutron- ${ }^{208} \mathrm{~Pb}$ optical potential taken from the parametrization of Madland [28], evaluated at the apropriate energy per nucleon, and $\rho_{n n}(r)$ is the neutron-neutron density distribution. The latter was calculated by integrating the square of the three-body wave function of the ${ }^{6} \mathrm{He}$ nucleus along the $2 n-\alpha$ coordinate, that is,

$$
\rho\left(r_{n n}\right)=r_{2 n-\alpha}^{2} \int\left|\Psi^{3 b}\left(\mathbf{r}_{n n}, \mathbf{r}_{2 n-\alpha}\right)\right|^{2} d \mathbf{r}_{2 n-\alpha} d \Omega_{n n},
$$

where $\Psi^{3 b}\left(\mathbf{r}_{n n}, \mathbf{r}_{2 n-\alpha}\right)$ is the three-body wave function and $\Omega_{n n}$ denotes the angular variables $\left(\theta_{n n}, \phi_{n n}\right)$. In the present calculations, the function $\Psi^{3 b}$ was taken from Ref. [24].

Very good convergence of the CDCC calculations was achieved with a basis of $N=30$ states. In addition, we found that continuum states above $50 \mathrm{MeV}$ have a negligible effect on the scattering observables, and hence these eigenstates were removed from the coupled-channels calculation. This reduces the number of PS included in the CDCC equations to $n_{s}=n_{p}=14$ and $n_{d}=15$ for $s, p$, and $d$ waves. The coupled equations were integrated up to $100 \mathrm{fm}$ and for a total angular momentum up to $J_{\max }=2000$.

In Fig. 7 we show the calculated energy differential breakup cross section, along with the GSI data. The dotted, dashed, and dotted-dashed lines are the separate contributions of the $s, p$, and $d$ continuum states. For this angular range $\left(\theta_{\text {lab }}<\right.$ $80 \mathrm{mrad}$ ) the cross section is largely dominated by the coupling

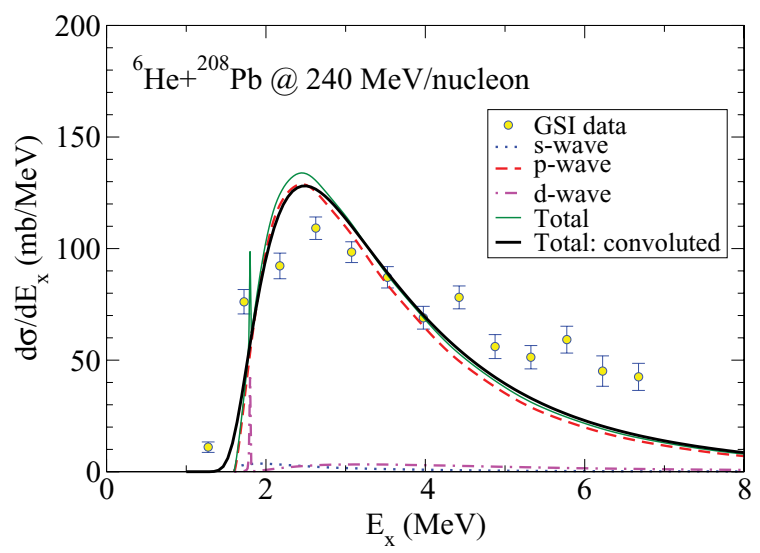

FIG. 7. (Color online) Angle-integrated breakup differential cross section, as a function of the excitation energy, for the reaction ${ }^{6} \mathrm{He}+{ }^{208} \mathrm{~Pb}$ at $240 \mathrm{MeV} /$ nucleon. The dotted, dashed, and dotteddashed lines are the contribution of the $s, p$, and $d$ states. The thin solid line is the sum of these contributions and the thick solid line is the full calculation convoluted with the experimental resolution. The circles are the data from Ref. [25]. to the $j=1^{-}$states due, mainly, to the strong dipole Coulomb interaction.

The thin solid line in Fig. 7 is the sum of the $s, p$, and $d$ contributions. For a meaningful comparison with the data, this curve has to be convoluted with the experimental energy resolution, which we took from the same work [25]. The result of this folding is shown by the thick solid line. At low excitation energies, this calculation reproduces very well the shape and magnitude of the data. For excitation energies $E_{x}>4 \mathrm{MeV}$, the calculation underpredicts the data. This discrepancy was also found in the semiclassical calculations reported in Ref. [25]. Note that the narrow peak due to the $2^{+}$resonance in ${ }^{6} \mathrm{He}$ disappears in the folded calculation.

The dominance of the dipole Coulomb couplings at these very small angles was used in Ref. [25] to extract the $d B(E 1) / d \varepsilon$ distribution, by comparing the measured differential cross section, $d \sigma / d E_{x}$, with semiclassical calculations. Although our calculations confirm the dominance of the E1 couplings, we have found that nuclear potentials have a small but not negligible effect on this observable. In addition, starting from the same structure model for the ${ }^{6} \mathrm{He}$ nucleus, our CDCC calculations show some departure from the semiclassical calculations, suggesting that the connection between the energy differential cross section and the underlying $E 1$ probability is more complicated than suggested by the semiclassical approach. These results are potentially very interesting because they may affect the extracted $d B(E 1) / d \varepsilon$ distribution from the cross section data. This analysis is beyond the scope of the present work and we leave it for a separate publication.

We consider now the ${ }^{12} \mathrm{C}$ target. The $2 n-\alpha$ interaction and the parameters of the LST were the same as those used in the $\mathrm{Pb}$ case. For the ${ }^{6} \mathrm{He}$ continuum states, $\ell=0-3$ waves were included. In this case, we used a THO basis with $N=40$ states. The $\alpha+{ }^{12} \mathrm{C}$ potential was adopted from Ref. [29]. For the $2 n+{ }^{12} C$ interaction we used the same single-folding model as for the ${ }^{208} \mathrm{~Pb}$ case taking also the neutron-target potential, $n+$ ${ }^{12} \mathrm{C}$ in this case, from the parametrization of Madland [28]. The coupled equations were integrated up to $100 \mathrm{fm}$ and for a total angular momentum up to $J_{\max }=200$. In Fig. 8 we compare the CDCC calculations with the experimental data from Ref. [25]. The meanings of the curves are the same as those in Fig. 7. In this case, the low-energy cross section is dominated by the population of the $2^{+}$continuum, with $s$ and $p$ waves contributing to the background. The contribution of the $f$ wave was found to be very small and hence it has not been included in this figure. As in the $\mathrm{Pb}$ case, the $d$-wave cross section shows a narrow peak corresponding to the well-known resonance at $E_{x}=1.8 \mathrm{MeV}$. The width of the peak in our calculation is significantly smaller than the experimental width of this resonance $\left(\Gamma_{\exp } \approx 100 \mathrm{keV}\right)$. This is a consequence of our simple two-body model adopted for the ${ }^{6}$ He nucleus. Nevertheless, when the calculation is convoluted with the experimental resolution (thick solid line) it becomes very close to the data. Despite the simplicity of the structure model adopted in these calculations, these results show that the THO discretization method constitutes a useful method to describe accurately detailed structures of the continuum that may show up in scattering observables. 


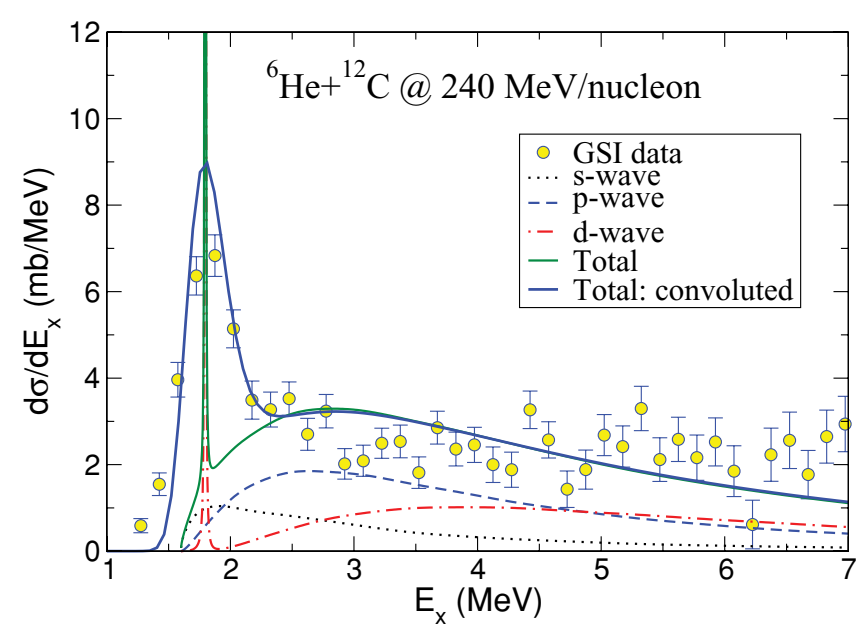

FIG. 8. (Color online) Energy breakup differential cross section, as a function of the excitation energy, for the reaction ${ }^{6} \mathrm{He}+{ }^{208} \mathrm{~Pb}$ at $240 \mathrm{MeV} /$ nucleon. The dashed line is the CDCC calculation with the THO discretization method. The solid line is the result of convoluting the CDCC calculation with the experimental resolution. The circles are the data from Ref. [25].

As a final remark on these results, we note that, at these energies, relativistic effects might affect the calculated observables. Some recent exploratory studies [30,31] have shown that these effects produce an increase of about $10 \%-$ $15 \%$ on the calculated breakup cross section for the ${ }^{8} \mathrm{~B}+{ }^{208} \mathrm{~Pb}$ and ${ }^{11} \mathrm{Be}+{ }^{208} \mathrm{~Pb}$ reactions at $250 \mathrm{MeV} /$ nucleon. These effects affect only the very small angles and are mostly due to the modification of the Coulomb potential. Therefore, we might expect a similar effect in our ${ }^{6} \mathrm{He}+{ }^{208} \mathrm{~Pb}$ case. For the ${ }^{6} \mathrm{He}+{ }^{12} \mathrm{C}$ case, we do not expect these effects to be important, because dynamical relativistic corrections to the nuclear interaction were found by the same authors to be negligible at these energies. These corrections refer to dynamical effects exclusively. Relativistic kinematics effects were included in the referred works, as well as in our calculations, by using the appropriate relativistic momentum. In any case, the aim of our work is to show the ability of the THO basis to describe the continuum of a two-body system, and so the emphasis of our study is more on the description of the structure, rather than on the reaction mechanism.

\section{B. Application to the ${ }^{11} \mathrm{Be}+{ }^{12} \mathrm{C}$ reaction at $67 \mathrm{MeV} /$ nucleon}

As a final example, we consider the scattering of the halo nucleus ${ }^{11} \mathrm{Be}$ on a carbon target. This reaction has been recently measured by Fukuda and collaborators at RIKEN [32], to extract information on the ${ }^{11} \mathrm{Be}$ continuum by measuring neutron- ${ }^{10} \mathrm{Be}$ coincidences following the projectile breakup. The angle-integrated differential cross section as a function of the relative energy between the outgoing neutron and ${ }^{10} \mathrm{Be}$ displays a structure dominated by a prominent resonance at $E_{x}=1.78 \mathrm{MeV}$. This resonance was interpreted as a $d_{5 / 2}$ neutron coupled to the ${ }^{10} \mathrm{Be}$ ground state. A second bump was also observed at $E_{x}=3.41 \mathrm{MeV}$, which was given a tentative assignment of $3 / 2^{+}$, with a small contribution of the $d_{3 / 2}$ wave coupled to ${ }^{10} \mathrm{Be}\left(0^{+}\right)$and a larger contribution of the ${ }^{10} \mathrm{Be}\left(2^{+}\right) \otimes v 2 s_{1 / 2}$ configuration. As in previous analyses of this reaction $[33,34]$, we use a two-body model of the projectile, ${ }^{11} \mathrm{Be}={ }^{10} \mathrm{Be}(\mathrm{g} . \mathrm{s}$. $)+n$, and hence those states based on the core excited states are absent from our model space.

In the present $\mathrm{CDCC}$ calculations, the neutron- ${ }^{10} \mathrm{Be}$ interaction was taken from Ref. [35]. This potential reproduces the separation energy of the ground state $\left(1 / 2^{+}\right)$and first excited state $\left(1 / 2^{-}\right)$and the position of the $5 / 2_{1}^{+}$resonance, assuming the configurations $2 s_{1 / 2}, 1 p_{1 / 2}$, and $d_{5 / 2}$, respectively. The ${ }^{11}$ Be continuum was described with a THO basis with $N=25$ states. The LST was generated with the parameters $b=2.4 \mathrm{fm}$ and $\gamma=3.6 \mathrm{fm}^{1 / 2}$. Continuum states with configurations $s_{1 / 2}$, $p_{1 / 2}, p_{3 / 2}, d_{3 / 2}$, and $d_{5 / 2}$ were considered. After diagonalization of the projectile Hamiltonian in this THO basis, only those eigenstates with excitation energies below $E<20 \mathrm{MeV}$ were included in the coupled-channels calculations, because the breakup cross section was found to be very small above this energy. This leaves about 10-11 eigenstates for each partial wave. Following Ref. [34], the $n+{ }^{12} \mathrm{C}$ and ${ }^{10} \mathrm{Be}+{ }^{12} \mathrm{C}$ potentials were taken from Refs. [36] and [37], respectively. The coupled-channels equations were integrated up to a matching radius of $R=90 \mathrm{fm}$ and for total angular momenta up to $J=350$.

In Fig. 9 we compare the experimental [32] and calculated energy differential cross sections as a function of the $n-{ }^{10} \mathrm{Be}$ relative energy. Both the data and the calculations correspond to the angular range $0^{\circ} \leqslant \theta_{\text {c.m. }} \leqslant 12^{\circ}$. The calculated contribution of each partial wave is shown. The symbols correspond to the contribution of specific pseudo-states, whereas the continuous lines are obtained convoluting the discrete $S$ matrices with the exact continuum states. The low-lying continuum is dominated by the $p_{3 / 2}$ and $d_{5 / 2}$ waves, with the latter being responsible for the resonant peak at $E_{x}=1.78 \mathrm{MeV}$. The dotted line is the sum of the different partial waves, and the thick solid line is the result of folding this full calculation with the instrumental resolution quoted in Ref. [32]. At energies

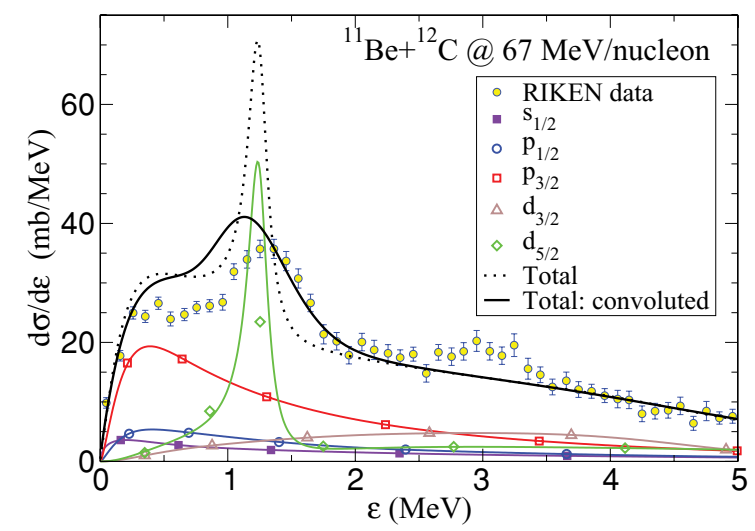

FIG. 9. (Color online) Energy breakup differential cross section, as a function of the excitation energy, for the reaction ${ }^{11} \mathrm{Be}+{ }^{12} \mathrm{C}$ at $67 \mathrm{MeV} /$ nucleon. The separate contribution of each partial wave is shown. The dotted line is the full contribution, and the thick solid line is the folding of the latter with the instrumental resolution. The experimental data are from Ref. [32]. 
close to the breakup threshold, the calculation overestimates the data. At energies above the resonance peak, the shape and magnitude of the data are well reproduced, with the exception of the broad peak due to the second resonance. We remind, however, that this resonance is believed to contain a significant contribution of ${ }^{10} \mathrm{Be}\left(2^{+}\right)$and, therefore, it is not expected to be well described with our model space. Our results are very close to those obtained by Howell et al. [34], including the overestimation of the data at low excitation energies. Because the calculations of that work used a continuum discretization in terms of energy bins, we attribute this discrepancy with the data to the choice of the interactions or to the restrictions of our three-body model, rather than to the method of discretization. As discussed in the ${ }^{6} \mathrm{He}+{ }^{12} \mathrm{C}$ case, an advantage of the THO discretization over the standard binning method is the ability of describing fine structures of the continuum with a relatively small basis. For example, to describe $d_{5 / 2}$ resonance, the CDCC calculations of Ref. [34] used 15 bins for $\varepsilon=0.5-$ $2 \mathrm{MeV}$, whereas in the present calculations about $10 \mathrm{PS}$ are enough to describe the full energy region, including the narrow resonance.

As in the ${ }^{6} \mathrm{He}$ case, for the $d_{5 / 2}$ continuum we get an eigenstate at $\varepsilon=1.25 \mathrm{MeV}$, which is close to the nominal energy of the resonance $(\varepsilon=1.27 \mathrm{MeV})$ and hence it is plausible to associate this eigenstate with the resonance structure. To corroborate this conclusion, in Fig. 10 we compare the experimental angular distribution of the resonance region [32] with the angular distribution of the three eigenstates closer to the nominal resonance energy. As anticipated, the eigenstate at $\varepsilon=1.25 \mathrm{MeV}$ reproduces fairly well the shape and magnitude of the data, supporting our conclusion that this eigenstate carries most of the resonant character. It has to be borne in mind that, as the basis size is increased, the resonant character will be distributed among several eigenstates and hence this identification is not possible. In fact, for the present basis size $(N=25)$ there might be some mixing between the three eigenstates shown in Fig. 10.

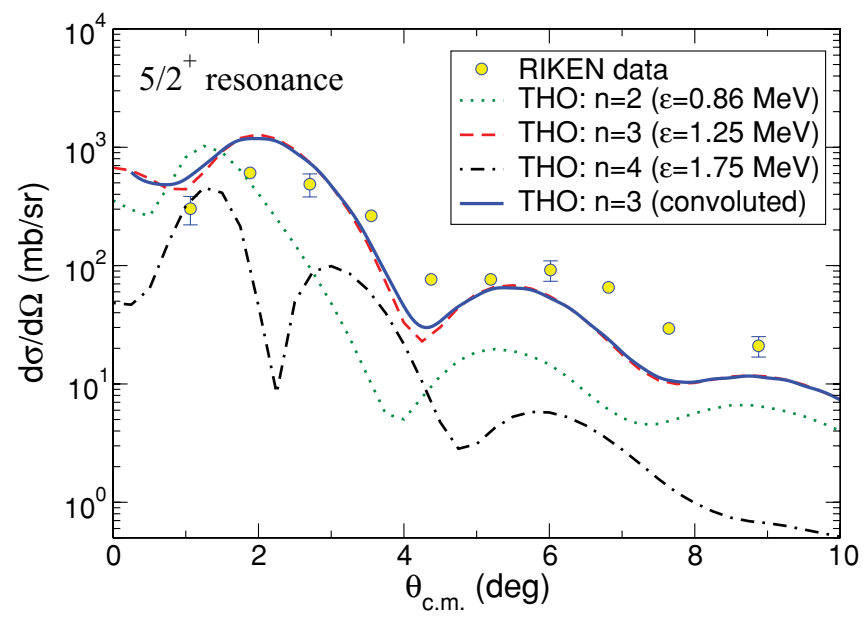

FIG. 10. (Color online) Angular distribution for the $E_{x}=$ 1.78 MeV resonance. The circles are the data points from Ref. [32]. The curves are the angular distribution due to the three PS around the resonance. See text for details.

\section{SUMMARY AND CONCLUSIONS}

In this work we have dealt with the problem of the study of the continuum properties of a weakly bound system in terms of basis of square-integrable functions, or PS. The general idea of the PS method is to diagonalize the Hamiltonian of the two-body system in a truncated PS basis. The eigenstates with negative energies represent the bound states of the system, whereas those at positive energies are regarded as a finite and discrete representation of the continuum spectrum. Among the many possible choices of the PS basis, in this work we have made use of the THO basis proposed in Refs. [6,11], in which the PS functions are generated by applying a parametric LST to the HO basis. The analytic form of the LST makes very simple the calculation of the PS basis. In addition, the radial extension of the basis and the energy distribution of the eigenvalues can be controlled through the parameters defining the LST. This permits one to adapt the properties of the basis to the problem at hand.

To test the accuracy of the THO basis to represent the continuum, we have evaluated the scattering phase-shifts for the deuteron and ${ }^{6} \mathrm{He}$ systems, treated as two-body systems $(p+n$ and $\alpha+2 n)$ interacting with a simple central interaction. Because the THO states vanish at large distances, the phase shifts have been evaluated using an integral formula, following the prescription of Hazi and Taylor [12]. In both cases, we find an excellent agreement with the exact phase shifts, obtained from the asymptotic part of the scattering states. Even the sharp resonance in the ${ }^{6} \mathrm{He}$ case is very well described with a small THO basis. As an additional test, we have evaluated the electric transition probabilities $B(E 1)$ and $B(E 2)$ for the same systems, finding again an excellent agreement with the results obtained with the scattering states. For this observable, a simple smoothing procedure has been proposed to provide a continuous distribution $(d B(E \lambda) / d \varepsilon)$ in terms of the discrete values obtained with the PS basis.

Finally, we have presented CDCC calculations for the reactions ${ }^{6} \mathrm{He}+{ }^{12} \mathrm{C}$ and ${ }^{6} \mathrm{He}+{ }^{208} \mathrm{~Pb}$ at $240 \mathrm{MeV} /$ nucleon and ${ }^{11} \mathrm{Be}+{ }^{12} \mathrm{C}$ at $67 \mathrm{MeV} /$ nucleon for which experimental data exist $[25,32]$. For the ${ }^{11} \mathrm{Be}+{ }^{12} \mathrm{C}$ reaction, we have used a two-body model ${ }^{10} \mathrm{Be}(\mathrm{g}$.s. $)+n$ of the projectile. To compare with the recent data of Fukuda et al. [32], we have calculated the breakup differential cross section as a function of the neutron- ${ }^{10} \mathrm{Be}$ relative energy. Using a relatively small THO basis, we have been able to reproduce fairly well the data, including the narrow $d_{5 / 2}$ resonance at $\varepsilon=1.27 \mathrm{MeV}$. Interestingly, one of the THO eigenstates appears at an energy very close to this energy and its associated differential angular cross section reproduces fairly well the experimental angular distribution obtained for the resonance region.

For the ${ }^{6} \mathrm{He}$ reactions, we have used a simple two-body model $(\alpha+2 n)$. Our calculations, which are parameter free, reproduce quantitatively and qualitatively the experimental energy differential cross sections reported in Ref. [25] both for the heavy target $\left({ }^{208} \mathrm{~Pb}\right)$ and for the light target $\left({ }^{12} \mathrm{C}\right)$. Furthermore, these calculations indicate that, for the ${ }^{6} \mathrm{He}+$ ${ }^{208} \mathrm{~Pb}$ reaction at excitation energies below $\sim 4 \mathrm{MeV}$, breakup cross sections are Coulomb dominated, with monopole and quadrupole components contributing only about $6 \%$. However, 
for ${ }^{6} \mathrm{He}+{ }^{12} \mathrm{C}$, the dominant component is the quadrupole, so that for excitation energies below $\sim 2.5 \mathrm{MeV}$, dipole and monopole components contribute about $24 \%$.

The role of nuclear forces and higher-order effects has been investigated by comparing our full coupled channels calculation with Coulomb calculations using the equivalent photon model. Differences as large as $28 \%$ have been found, indicating the need for performing CDCC calculations to extract structure information from breakup reaction data, at least in this energy regime.

The agreement between theory and experiment is very encouraging, given the simplicity of the dineutron model used in the present calculations. One has to bear in mind, however, that some of the details of the breakup distributions might be hidden due to the energy resolution of the experiment. New measurements with better energy resolution will be useful to test more stringently the breakup distributions at energies closer to the threshold. An accurate description of these reactions will require a realistic three-body model to describe the ${ }^{6} \mathrm{He}$ nucleus. In this respect, it is worth noting that the THO method used in this work can be generalized to three-body problems. This can provide a useful yet simple method to study continuum structures (e.g., resonances) in nuclei with a three-body structure $\left({ }^{9,14} \mathrm{Be},{ }^{6} \mathrm{He},{ }^{11} \mathrm{Li},{ }^{8} \mathrm{~B}\right.$, etc. $)$ as well as reactions involving these nuclei. A similar approach proposed very recently, making use of a different PS basis, has been found to provide very promising results $[38,39]$.

\section{ACKNOWLEDGMENTS}

We are grateful to T. Aumann for supplying us the experimental data in tabular form and for his help interpreting these data. This work has been partially supported by the Spanish Ministerio de Ciencia e Innovación under Projects FIS2008-04189, FPA2009-07653, and FPA2009-08848 and by the Spanish Consolider-Ingenio 2010 Programme CPAN (CSD2007-00042). J.A.L. acknowledges a research grant from the Ministerio de Ciencia e Innovación.
[1] R. C. Johnson and P. J. R. Soper, Phys. Rev. C 1, 976 (1970).

[2] G. H. Rawitscher, Phys. Rev. C 9, 2210 (1974).

[3] N. Austern, Y. Iseri, M. Kamimura, M. Kawai, G. Rawitscher, and M. Yahiro, Phys. Rep. 154, 125 (1987).

[4] T. Matsumoto, T. Kamizato, K. Ogata, Y. Iseri, E. Hiyama M. Kamimura, and M. Yahiro, Phys. Rev. C 68, 064607 (2003).

[5] F. Pérez-Bernal, I. Martel, J. M. Arias, and J. Gómez-Camacho, Few-Body Syst. Suppl. 13, 217 (2002).

[6] A. M. Moro, J. M. Arias, J. Gómez-Camacho, and F. PérezBernal, Phys. Rev. C 80, 054605 (2009).

[7] T. Matsumoto, E. Hiyama, M. Yahiro, K. Ogata, Y. Iseri, and M. Kamimura, Nucl. Phys. A 738, 471 (2004).

[8] T. Matsumoto, E. Hiyama, K. Ogata, Y. Iseri, M. Kamimura, S. Chiba, and M. Yahiro, Phys. Rev. C 70, 061601(R) (2004).

[9] T. Matsumoto, T. Egami, K. Ogata, Y. Iseri, M. Kamimura, and M. Yahiro, Phys. Rev. C 73, 051602(R) (2006).

[10] M. Rodríguez-Gallardo, J. M. Arias, J. Gómez-Camacho, R. C. Johnson, A. M. Moro, I. J. Thompson, and J. A. Tostevin, Phys. Rev. C 77, 064609 (2008).

[11] S. Karataglidis, K. Amos, and B. G. Giraud, Phys. Rev. C 71, 064601 (2005).

[12] A. U. Hazi and H. S. Taylor, Phys. Rev. A 1, 1109 (1970).

[13] K. Chadan, R. Kobayashi, and T. Kobayashi, J. Math. Phys. 42, 4031 (2001).

[14] Y. Suzuki, W. Horiuchi, and K. Arai, Nucl. Phys. A 823, 1 (2009).

[15] A. U. Hazi and M. F. Fels, Chem. Phys. Lett. 8, 582 (1971).

[16] H. S. Taylor and A. U. Hazi, Phys. Rev. A 14, 2071 (1976).

[17] M. V. Stoitsov and I. Z. Petkov, Ann. Phys. (NY) 184, 121 (1988).

[18] I. Z. Petkov and M. V. Stoitsov, Nuclear Density Functional Theory, Oxford Studies in Physics (Clarendon, Oxford, 1991).

[19] F. Pérez-Bernal, I. Martel, J. M. Arias, and J. Gómez-Camacho, Phys. Rev. A 63, 052111 (2001).

[20] S. Typel and G. Baur, Nucl. Phys. A 759, 247 (2005).
[21] A. M. Moro, J. M. Arias, J. Gómez-Camacho, I. Martel, F. Pérez-Bernal, R. Crespo, and F. M. Nunes, Phys. Rev. C 65, 011602(R) (2001).

[22] C. J. Joachain, Quantum Collision Theory (North-Holland, Amsterdam, 1987).

[23] J. L. Friar, S. Fallieros, E. L. Tomusiak, D. Skopik, and E. G. Fuller, Phys. Rev. C 27, 1364 (1983).

[24] A. M. Moro, K. Rusek, J. M. Arias, J. Gómez-Camacho, and M. Rodríguez-Gallardo, Phys. Rev. C 75, 064607 (2007).

[25] T. Aumann et al., Phys. Rev. C 59, 1252 (1999).

[26] J. A. Tostevin, F. M. Nunes, and I. J. Thompson, Phys. Rev. C 63, 024617 (2001).

[27] B. Bonin et al., Nucl. Phys. A 445, 381 (1985).

[28] D. G. Madland, in Proceedings of OECD/NEA Specialists' Meeting on the Nucleon-Nucleus Optical Model up to $200 \mathrm{MeV}$ (NEA/OECD, Paris, 1997), p. 129.

[29] D. T. Khoa, G. R. Satchler, and N. D. Thuy, Phys. Rev. C 65, 024611 (2002).

[30] K. Ogata and C. A. Bertulani, Prog. Theor. Phys. (Kyoto) 121, 1399 (2009).

[31] K. Ogata and C. A. Bertulani, Prog. Theor. Phys. (Kyoto) 123, 701 (2010).

[32] N. Fukuda et al., Phys. Rev. C 70, 054606 (2004).

[33] P. Capel, G. Goldstein, and D. Baye, Phys. Rev. C 70, 064605 (2004).

[34] D. J. Howell, J. A. Tostevin, and J. S. Al-Khalili, J. Phys. G 31, S1881 (2005).

[35] E. Cravo, R. Crespo, A. M. Moro, and A. Deltuva, Phys. Rev. C 81, 031601 (2010).

[36] F. D. Becchetti and G. W. Greenlees, Phys. Rev. 182, 1190 (1969).

[37] J. S. Al Khalili, J. A. Tostevin, and J. M. Brooke, Phys. Rev. C 55, R1018 (1997).

[38] P. Barletta, C. Romero-Redondo, A. Kievsky, M. Viviani, and E. Garrido, Phys. Rev. Lett. 103, 090402 (2009).

[39] A. Kievsky, M. Viviani, P. Barletta, C. Romero-Redondo, and E. Garrido, Phys. Rev. C 81, 034002 (2010). 